\title{
The relation between plasma levels of nesfatin- 1 and different grades of diabetic kidney disease in patients with type 2 diabetes
}

\author{
Mohamed A. Korani ${ }^{\mathrm{a}}$, Ahmed Sonbol ${ }^{\mathrm{b}}$
}

Departments of a Internal Medicine ${ }^{b}$ Clinical Pathology, Menoufia University, Shebeen El-Kom, Egypt

Correspondence to Mohamed A. Korani, Assistant Professor of Internal Medicine Department, Faculty of Medicine, Menoufia University, Egypt. Tel: +20 1010903937 . e-mail: mohammedelsayed29@icloud.com

Received 12 November 2017 Accepted 30 January 2018

The Egyptian Journal of Internal Medicine 2018, 30:68-71

\begin{abstract}
Background
Diabetic kidney disease (DKD) is the leading cause of renal failure. Diabetic patients with microalbuminuria typically progress to proteinuria and overt diabetic nephropathy. Nesfatin-1 is an anoretic polypeptide with potent metabolic regulatory effects and its circulating levels are shown to be elevated in type 2 diabetes mellitus (T2 DM).

Nesfatin-1 may be involved in the pathogenesis of DKD through inflammatory mechanisms.

The aim of the work was to study the relation between plasma nesfatin- 1 levels and different grades of DKD in patients with T2 DM.

Patients and methods

This study was conducted on 120 patients with T2 DM and 20 controls selected from the inpatient department and outpatient clinics of the Internal Medicine Department in Menoufia University Hospital. The selected individuals were grouped into four groups: group 1 included 20 healthy individuals, group 2 included 40 patients with T2 DM with normoalbuminuria, group 3 included 40 patients with T2 DM with microalbuminuria, and group 4 included 40 patients with T2 DM with macroalbuminuria.

Members of the study were subjected to thorough history with special emphasis on age, sex, and duration of the DM. Investigations included fasting blood glucose, glycosylated hemoglobin, complete urine analysis, serum creatinine, urine albumin-creatinine ratio, estimated glomerular filtration rate, and plasma nesfatin-1. Results

Plasma nesfatin- 1 was significantly higher in group 4 compared with other groups, in group 3 compared with groups 1 and 2 , and in group 2 compared with group 1 . There was a positive significant correlation between plasma nesfatin-1 and serum creatinine, urine albumin-creatinine ratio, and glycosylated hemoglobin and negative significant correlation between plasma nesfatin-1 and estimated glomerular filtration rate in the studied diabetic patients. There was no correlation between plasma nesfatin-1 and BMI and duration of diabetes in the diabetic patients.

Conclusion

From this study, we can conclude that plasma nesfatin- 1 is significantly higher in patients with DKD, and there is a positive correlation between plasma nefatin-1 level and the grades of DKD. So, plasma nesfatin-1 may be considered for prediction of developing DKD in a previously unaffected patients with DM.
\end{abstract}

\section{Keywords:}

diabetic kidney disease, plasma nesfatin-1, type 2 diabetes mellitus

Egypt J Intern Med 30:68-71

(C) 2018 The Egyptian Journal of Internal Medicine

$1110-7782$

\section{Introduction}

Nesfatin-1 is an 82 amino-acid length polypeptide derived from calcium and DNA-binding protein NUCB2 [1].

The nesfatin-1 is promimently expressed in several regions of the hypothalamus nuclei. Moreover, it is expressed in several peripheral tissues including pancreatic $\beta$ cells. Plasma levels of nesfatin-1 are reduced by fasting, and increased after refeeding, and this suggests that nesfatin-1 plays a role in satiety regulation and, possibly, energy homeostasis [2].
Nesfatin-1 crosses the blood-brain barrier in both the blood-to-brain and brain-to-blood directions by nonsaturable mechanisms [3].

A research study has shown an antihyperglycemic effect of nesfatin-1. The results obtained indicate

This is an open access journal, and articles are distributed under the terms of the Creative Commons Attribution-NonCommercial-ShareAlike 4.0 License, which allows others to remix, tweak, and build upon the work non-commercially, as long as appropriate credit is given and the new creations are licensed under the identical terms. 
that the antihyperglycemic effect of nesfatin-1 is peripheral and dependent on time, dose, and insulin.

Experimental studies have also linked nesfatin-1 to enhanced hepatic and peripheral insulin sensitivity by promoting peripheral glucose uptake and decreasing gluconeogenesis through different mechanisms $[4,5]$.

Nesfatin-1 has been studied in several metabolic dysregulations such as obesity, inflammation, and diabetes $[6,7]$.

Diabetic kidney disease (DKD) is a chronic microvascular complication of diabetes that may lead to end-stage renal disease [8]. Early diagnosis and effective treatment of DKD may delay the progression of this disease [9]. So, reliable screening biomarker of DKD would be of value and an important issue in detecting the patients in need for further assessment and treatment. We presumed that nesfatin-1 may play a role in the development of DKD.

The aim of the work was to study the relation between plasma nesfatin-1 levels and different grades of DKD in type 2 diabetes mellitus (T2 DM).

\section{Patients and methods}

This study was conducted on 120 patients with T2 DM selected from the inpatient department and outpatient clinics of the Internal Medicine Department during the period from June 2017 to November 2017. There were 40 patients with normoalbuminuria and 80 patients with DKD. DKD was confirmed by exclusion of the other conditions that may affect the kidneys through full history and clinical examination to exclude uncontrolled hypertension, chronic inflammatory conditions, malignancy, repeated urinary tract infection; through investigations including urine analysis to exclude the presence of significant urinary casts (red cell casts) and urinary tract infection; and ultrasound of the kidneys to exclude polycystic kidney disease, obstructive uropathy, renal stones, and renal artery stenosis.

In addition, 20 healthy individuals were included in the control group. The protocol of this study was approved by the Ethical Committee of Faculty of Medicine.

The selected participants were grouped into four groups:

Group 1: it included 20 healthy individuals as a control group.
Group 2: it included 40 T2 DM with normoalbuminuria.

Group 3: it included 40 T2 DM with microalbuminuria.

Group 4: it included 40 T2 DM with macroalbuminuria.

\section{Exclusion criteria}

The exclusion criteria were type $1 \mathrm{DM}$, severe hepatic impairment, end-stage renal disease, uncontrolled hypertension, chronic inflammatory disease, malignancy, acute infections, and nephropathy owing to causes other than diabetes.

After taking their consent for participation in the study, all members of the study were subjected to thorough history with special emphasis on age, sex, duration of DM, and complete physical examination. Investigations included fasting blood glucose, glycosylated hemoglobin, fasting serum insulin, serum creatinine, serum alanine transaminase, urine analysis, urine albumin-creatinine ratio, and plasma nesfatin-1 concentration were measured using human enzyme-linked immunosorbent assay kits, human nesfatin-1 PicoKine ELISA Kit (Boster Biological Technology, Pleasanton, California, USA). Glomerular filtration rate was estimated by CockroftGault formula [10].

Assessment of insulin resistance was done by using homeostasis model assessment $2\left(\mathrm{HOMA}_{2}\right)$ calculator according to the updated computer-based $\mathrm{HOMA}_{2}$ model in patients with normal and impaired glucose tolerance [11].

\section{Statistical methodology}

Data were analyzed using IBM SPSS software package, version 20.0 (Armonk, NY: IBM Crop Chicago, USA).

Qualitative data were presented as frequency and percentage. Quantitative data were presented as mean and SD. To compare between groups, we used $\chi^{2}$-test, analysis of variance test, and least significant difference and correlation coefficient. Significance level value was $P$ equal to or less than 0.05 .

\section{Results}

There was no significant difference between the studied groups regarding age and sex. BMI was significantly higher in diabetic groups compared with the control group. The duration of DM was significantly higher in group 4 compared with groups 2 and 3. 
Glycosylated hemoglobin was significantly higher in group 4 compared with other diabetic groups. Urine albumin-creatinine ratio (UACR) was significantly higher in group 4 compared with other groups and in group 3 compared with groups 2 and 1. The estimated glomerular filtration rate (eGFR) was significantly lower in group 4 compared with other groups and in group 3 compared with groups 1 and 2 (Table 1).

Plasma nesfatin-1 level was significantly higher in diabetic groups compared with control group and in group 4 compared with other groups and in group 3 compared with groups 1 and 2 , and in group 2 compared with group 1 (Table 2).

There was a positive significant correlation between plasma nesfetin-1 and serum creatinine, glycosylated hemoglobin (HbA1c), and UACR, and a negative significant correlation between plasma nesfatin-1 and eGFR in the diabetic patients. There was no correlation between plasma nesfatin-1 and BMI and duration of diabetes (Table 3).

\section{Discussion}

The pathogenesis of DKD is multifactorial. Therefore, to study the relation between nesfatin- 1 and DKD, we study also its relation to BMI, duration of diabetes, glycemic control, and insulin resistance.

In the current study, plasma nesfatin-1 was significantly higher in diabetic patients compared with the nondiabetic individuals; moreover, it is significantly higher in macroalbuminuric than microalbuminuric and normoalbuminuric patients.
There was a positive correlation between the nesfatin- 1 and serum creatinine and UACR, whereas there was a negative correlation between nesfatin-1 and eGFR.

Although plasma nesfatin-1 was shown to be increased in patients with diabetes [12], conflicting results have refuted the presence of such association [13].

Possible inflammatory mechanisms should be considered to explain the serum nesfatin-1 increased levels in patient with DKD. Albuminuria actively contributes to endothelial dysfunction that is predominantly characterized by long-term and lowgrade state of systemic inflammation. At the level of hypothalamus and brain stem, a proportion of nesfatin1-producing neurons are highly sensitive to peripheral inflammatory stimuli $[14,15]$.

Elevated plasma nesfatin- 1 is also because of activation of mechanisms against DKD-mediated metabolic and inflammatory disturbance. Consistently, Joing et al. [14] demonstrated the potency of nesfatin-1 as a potential therapeutic agent in renal ischemic reperfusion injury through its anti-inflammatory, antioxidant, and antiapoptotic effect. Nesfatin-1 has also an anti-inflammatory feature, especially in brain damage $[15,16]$.

Recent histopathologic studies reported the overexpression levels of $\mathrm{N} 4 \mathrm{CB} 2$ nesfatin-1 and binding sites localized to the renal tissue cells at both mRNA and protein levels in the absence of inflammation. These reports [17] suggest that the increase in plasma NUCB2 nesfatin-1 is derived from the renal tubules in DKD that acts as one of renal protective mechanisms [18].

Table 1 Comparison between the studied groups according to demographic and laboratory investigations

\begin{tabular}{lccccccc}
\hline & $\mathrm{G} 1(n=20)$ & $\mathrm{G} 2(n=40)$ & $\mathrm{G} 3(n=40)$ & $\mathrm{G} 4(n=40)$ & $F$-test & $P$ & \multicolumn{1}{c}{ LSD } \\
\hline Age (years) & $55.0 \pm 7.6$ & $54.6 \pm 8.1$ & $53.9 \pm 6.1$ & $55.5 \pm 7.1$ & 0.342 & 0.795 & - \\
Duration of diabetes (years) & - & $6.1 \pm 1.7$ & $7.3 \pm 2.3$ & $10.1 \pm 2.1$ & 21.732 & $<0.001^{*}$ & 2 vs. 3.43 vs. 4 \\
BMI $\left(\mathrm{kg} / \mathrm{m}^{2}\right)$ & $24.034 \pm 1.28$ & $27.18 \pm 2.01$ & $29.28 \pm 3.54$ & $31.19 \pm 4.02$ & 14.443 & $<0.001^{*}$ & 2 vs. 3.43 vs. 4 \\
HbAlc $(\%)$ & $5.51 \pm 0.29$ & $6.9 \pm 0.9$ & $7.86 \pm 1.08$ & $9.03 \pm 1.85$ & 22.296 & $<0.001^{*}$ & 2 vs. 3.43 vs. 4 \\
HOMA & $2.03 \pm 0.91$ & $4.33 \pm 0.88$ & $7.70 \pm 6.0$ & $10.04 \pm 7.21$ & 14.934 & $<0.001^{*}$ & 1 vs. 3.42 vs. 3.43 vs. 4 \\
Creatinine & $0.66 \pm 0.12$ & $1.06 \pm 0.17$ & $1.5 \pm 0.18$ & $2.4 \pm 0.65$ & 128.451 & $<0.001^{*}$ & 1 vs. 3.42 vs. 3.43 vs. 4 \\
UACR $(\mathrm{mg} / \mathrm{gcv})$ & - & $20.1 \pm 3.6$ & $209.7 \pm 31.06$ & $1201 \pm 206.5$ & 1106.443 & $<0.001^{*}$ & 2 vs. 3.43 vs. 4 \\
eGFR $\left(\mathrm{ml} / \mathrm{min} / 1.73 \mathrm{~m}^{2}\right)$ & $114.4 \pm 7.6$ & $97.61 \pm 5.6$ & $77.6 \pm 6.4$ & $41.3 \pm 5.7$ & 837.219 & $<0.001^{*}$ & 1 vs. 3.42 vs. 3.43 vs. 4 \\
\hline
\end{tabular}

eGFR, estimated glomerular filtration rate; HbAlc, glycosylated hemoglobin; HOMA, homeostasis model assessment for insulin resistance; LSD, least significant difference; UACR, urine albumin-creatinine ratio; "Statistically significant at $P \leq 0.05$.

Table 2 Comparison between the studied groups regarding plasma nesfatin-1 levels

\begin{tabular}{llllllll}
\hline & $\mathrm{G} 1(n=20)$ & $\mathrm{G} 2(n=40)$ & $\mathrm{G} 3(n=40)$ & $\mathrm{G} 4(n=40)$ & $F$-test & $P$ & LSD \\
\hline Plasma nesfatin-1 levels $(\mathrm{pg} / \mathrm{ml})$ & $0.41 \pm 0.94$ & $2.03 \pm 1.01$ & $3.04 \pm 1.99$ & $4.88 \pm 2.04$ & 41.894 & $<0.001^{*}$ & 1 vs. 3.42 vs. 3.43 vs. 4 \\
\hline
\end{tabular}

* Statistically significant at $P \leq 0.05$. 
Table 3 Correlation between the plasma nesfatin-1 and other parameters in the diabetic patients

\begin{tabular}{lcc}
\hline & \multicolumn{2}{c}{ Serum nesfatin-1 } \\
\cline { 2 - 3 } & $r$ & $P$ value \\
\hline Duration of diabetes & 0.135 & 1.141 \\
Serum creatinine & 0.640 & $<0.001^{*}$ \\
Urine albumin-creatinine ratio & 0.511 & $<0.001^{*}$ \\
Estimated glomerular filtration rate & -0.607 & $<0.001^{*}$ \\
Glycosylated hemoglobin & 0.576 & $<0.001^{*}$ \\
BMI & 0.154 & 0.059 \\
Homeostasis model assessment of insulin & 0.68 & $<0.001^{*}$
\end{tabular}

resistance

$r$, correlation coefficient; * Statistically significant at $P \leq 0.05$.

In our study, there was a positive correlation between nesfatin-1 and homeostasis model assessment of insulin resistance, which is in agreement with Zhang et al. [12]. They confirmed a positive correlation between nesfatin-1 with fasting plasma insulin and HOMA. Moreover, there was a positive correlation between nesfatin-1 and $\mathrm{HbA} 1 \mathrm{c}$, which is in agreement with Ademoglu et al. [19], who found plasma nesfatin1 to be positively associated with $\mathrm{HbA} 1 \mathrm{c}$. Other groups found nesfatin-1 and $\mathrm{HbA} 1 \mathrm{c}$ were negatively correlated in hypothyroid patients [20]. Increased level of $\mathrm{HbA} 1 \mathrm{c}$ and poor glycemic control act as an inflammatory milieu in the kidney and stimulate protein kinase-c and oxidative stress, which increases the expression of cytokines with more attraction of inflammatory cells to the kidney, which in turn lead to endothelial dysfunction and albuminuria.

\section{Conclusion}

Plasma nesfatin-1 levels are significantly elevated in patients with T2 DM and DKD, and such levels of elevation are related to the severity of DKD. Owing to the highly significant statistical difference in plasma nesfatin-1 levels between diabetics with normoalbuminuria and diabetics with albuminuria, it has been known that some of patients with DKD are normoalbuminuric, so plasma nesfatin-1 level can add a predictive value in diagnosis of DKD in previously unaffected patients with diabetes.

\section{Financial support and sponsorship}

Nil.

\section{Conflicts of interest}

There are no conflicts of interest.

\section{References}

1 Oh-I S, Shimizu H, Satoh T. Identification of nesfatin-1 as a satiety molecule in the hypothalamus. Nature 2006; 443:709-712.

2 Abaci A, Catli G, Anik A, Kume T, Bober E. The relation of serum nesfatin-1 level with metabolic and clinical parameters in obese and healthy children. Pediatr Diab 2013; 14:189-195.

3 Pan W, Hsuchou H, Kastin AJ. Nesfatin-1 crosses the blood-brain barrier without saturation. Peptides 2007; 28:2223-2228.

4 Dong J, Xu H, Xu H, Wang PF, Cai GJ, Song HF. Nesfatin-1 stimulates fatty-acid oxidation by activating AMP-activated protein kinase in STZ-induced type 2 diabetic mice. PLoS One 2013; 8:833-863.

5 Wu D, Yan M, Chen Y, Jia Y, Ma ZA, Boden G, et al. Hypothalamic nesfatin1/NUCB2 knockdown augments hepatic gluconeogenesis that is correlated with inhibition of mTOR-STAT3 signaling pathway in rats. Diabetes 2014; 63:1234-1247.

6 Aydin S. Multi-functional peptide hormone NUCB2/nesfatin-1. Endocrine 2013; 44:312-325.

7 Aslan M, Celik O, Celik N, Turkcuoglu I, Yilmaz E, Karaer A, et al. Cord blood nesfatin-1 and apelin-36 levels in gestational diabetes mellitus. Endocrine 2012; 41:424-429.

8 Tuttle KR, Bakris GL, Bilous JL, Chiang H, Boer J, Goldstein F, et al. Diabetic kidney disease: a report from an ADA Consensus Conference. Am $J$ Kidney Dis 2014; 64:510-533.

9 Kathrine MD. The evaluation of diabetic nephropathy: preventing complications. Am J Kidney Dis 2002; 39:s1-s46.

10 Lewis J, Agodoa L, Cheek D, Greene T, Middleton J, O'Connor D, et al. Comparison of cross-sectional renal function measurements in African Americans with hypertensive nephrosclerosis and of primary formulas to estimate glomerular filtration rate. Am J Kidney Dis 2001; 38 744-753.

11 The University of Oxford. HOMA calculator; 2016. Available at: https://www. dtu.ox.ac.uk/homacalculator/. [Accessed 8 October 2016]

12 Zhang Z, Li L, Yang G, Liu G, Boden G, Yang M, et al. Increased plasma levels of nesfatin-1 in patients with newly diagnosed type 2 diabetes mellitus. Exp Clin Endocrinol Diab 2012; 120:91-95.

13 Li Q, Wang Y, Chen X, Guan Z, Jiang Y. Fasting plasma levels of nesfatin-1 in patients with type 1 and type 2 diabetes mellitus and the nutrient-related fluctuation of nesfatin-1 level in normal humans. Regul Pept 2010; 159: 72-77.

14 Jiang G, Wang M, Wang L, Chen H, Chen Z, Guo J, et al. The protective effect of nesfatin-1 against renal ischemia-reperfusion injury in rats. Ren Fail 2014; 37:882-889.

15 Özsavcí D, Erşahin M, Şener A, Özakpinar ÖB, Toklu HZ, Akakín D, et al The Novel Function of nesfatin-1as an anti-inflammatory and antiapoptotic peptide in subarachnoid hemorrhage-induced oxidative brain damage in rats. Neurosurgery 2011; 68:1699-1708

16 Anwar GM, Yamamah G, Ibrahim A, El-Lebedy D, Farid TM, Mahmoud R, et al. Nesfatin-1 in childhood and adolescent obesity and its association with food intake, body composition and insulin resistance. Regul Pept 2014; 188:21-24

17 Prinz P, Goebel-Stengel M, Teuffel P, Rose M, Klapp BF, Stengel A, et al Peripheral and central localization of the nesfatin-1 receptor using autoradiography in rats. Biochem Biophys Res Commun 2016; 470: $521-527$

18 Qi C, Ma H, Zhang HT, Gao JD, Xu Y Nucleobindin 2 expression is an independent prognostic factor for clear cell renal cell carcinoma. Histopathology 2016; 66:650-657.

19 Ademoglu EN, Gorar S, Carlıoglu A, Yazic1 H, Dellal FD, Berberoglu Z, et al. Plasma nesfatin-1 levels are increased in patients with polycystic ovary syndrome. J Endocrinol Invest 2014; 37 715-719.

20 Liu F, Yang Q, Gao N, Liu S, Shen F. Decreased plasma nesfatin-1 level is related to the thyroid dysfunction in patients with type 2 diabetes mellitus. $J$ Diab Res 2014; 67:1739-1742. 